\title{
Spontaneous Breathing Trials in Patients Managed with a Sedation Protocol or a Sedation Protocol with Daily
}

\section{Interruption}

\author{
Rose $\mathbf{L}^{1,2,3,4}$, Burns KEA ${ }^{3,4,5}$, Dodek $\mathrm{P}^{6,7}$, Mallick $\mathbf{R}^{8,9}$, Fergusson $\mathrm{D}^{8,9}$, \\ Cook DJ10,11, Burry LD12,14, Meade $\mathbf{M}^{11,13}$ and Mehta $S^{* 3,15}$ \\ ${ }^{1}$ Department of Critical Care Medicine, Sunnybrook Health Sciences Centre, Toronto, \\ ON, Canada
}

Short communication

Volume 1 Issue 2

Received Date: August 22, 2016

Published Date: September 1, 2016

${ }^{2}$ Lawrence S. Bloomberg Faculty of Nursing, University of Toronto, Toronto, ON, Canada

${ }^{3}$ Interdepartmental Division of Critical Care Medicine, University of Toronto, Toronto, ON, Canada

${ }^{4} \mathrm{Li}$ Ka Shing Institute, Toronto, ON, Canada

${ }^{5}$ Division of Critical Care, St. Michael's Hospital, Toronto, ON, Canada

${ }^{6}$ Division of Critical Care Medicine and, St. Paul's Hospital

${ }^{7}$ Center for Health Evaluation and Outcome Sciences, University of British Columbia, Vancouver, BC, Canada.

${ }^{8}$ Clinical Epidemiology Program, Ottawa Hospital Research Institute, Ottawa, ON, Canada

${ }^{9}$ Faculty of Medicine, University of Ottawa, Ottawa, ON, Canada

${ }^{10}$ Department of Critical Care Medicine, St Joseph's Healthcare, Hamilton, ON, Canada

${ }^{11}$ Departments of Medicine, Clinical Epidemiology \& Biostatistics, McMaster University, Hamilton, ON, Canada

${ }^{12}$ Leslie Dan Faculty of Pharmacy, University of Toronto, Toronto, ON, Canada

${ }^{13}$ Department of Critical Care, Hamilton Health Sciences, Hamilton, ON, Canada

${ }^{14}$ Department of Pharmacy, Mt. Sinai Hospital

${ }^{15}$ Department of Critical Care Medicine, Mt. Sinai Hospital

*Corresponding author: Dr. S. Mehta Mount Sinai Hospital, 600 University Ave, RM 18-216, Toronto, ON Canada M5G 1X5, Tel: 416-586-4800/4604; Fax: 416-586-8480; E-mail: Geeta.mehta@utoronto.ca

\section{Abstract}

Rationale: Spontaneous breathing trials (SBT) allow evaluation of a patient's potential to breathe independently, but do not directly assess extubation readiness.

Objectives: To describe adherence to SBT screening and conduct, and extubation rates after successful SBTs in patients enrolled in a multicenter randomized trial comparing protocolized sedation with protocolized sedation plus daily sedation interruption. 


\section{Anaesthesia \& Critical Care Medicine Journal}

Methods: We recruited 430 critically ill, mechanically ventilated adults from 16 North American ICUs. In both study groups, patients were screened daily using standardized criteria to undergo an SBT; eligible patients underwent a 60minute SBT. If SBT was successful, respiratory therapists notified the clinical ICU team responsible for extubation decisions. If not performed, or no extubation despite passing an SBT, respiratory therapists documented 1 of 8 reasons.

Measurements and Main Results: SBT screening occurred on $92 \%$ and $93 \%$ days in the interruption and control groups, respectively. Among screened patients, interruption group was eligible for an SBT less often than controls $(32 \%$ versus $36 \%$ days, $\mathrm{P}=0.003$ ). In both groups, SBTs were performed on $82 \%$ of days SBTs were indicated. Despite successful SBT, extubation did not occur on $61 \%$ (interruption) and $65 \%$ (control) of days $(\mathrm{P}=0.24)$. Reasons for no extubation on the day of a successful SBT differed between groups; however the most common reasons in both groups were concerns about consciousness level and physician request.

Conclusion: Mechanically ventilated patients were extubated less than $40 \%$ of the time on the day of a successful SBT. The patterns, predictors and outcomes associated with delayed extubation warrant further evaluation. ClinicalTrials.gov NCT 00675363.

Keywords: Spontaneous breathing trial; Extubation; Mechanical ventilation; Sedation

\section{Introduction}

The routine application of spontaneous breathing trials (SBT) can reduce mechanical ventilation duration and hospital mortality [1,2]. SBTs, which comprise 30 to 120 minutes of minimal support via the ventilator, T-tube circuit, or tracheotomy mask, allow clinicians to evaluate a patient's potential to breathe independently, but do not directly assess extubation readiness [3]. In the ABC trial, addition of daily sedation interruption (DI) to routine SBTs demonstrated reductions in the durations of mechanical ventilation and intensive care unit (ICU) and hospital length of stay [4]. However, extubation decisions were not protocolized, and only $46 \%$ patients were extubated on the first day that they passed an SBT [4]. We report on the use of SBTs in the SLEAP trial, which compared two sedation minimization strategies, a sedation protocol alone (control group) versus sedation protocol plus DI [5]. Both groups underwent daily standardized screening by respiratory therapists for the potential to conduct a SBT. There was no difference in the primary outcome of time to successful extubation between the two groups.

\section{Objective}

To describe adherence to SBT screening and conduct, and the rates of extubation after successful SBTs in patients enrolled in the SLEAP trial.

\section{Methods}

This was a secondary analysis of the SLEAP trial, which was conducted in 16 tertiary ICUs in Canada and the United States. The complete SLEAP trial methods relating to study participants, the nurse-directed sedation protocol, and daily sedation interruption have been published [5]. Before the SLEAP trial, 14 ICUs routinely employed SBTs as the standard of care to guide extubation decisions; and in 2 ICUs SBT use varied by clinician. During the SLEAP trial, respiratory therapists assessed extubation-readiness in a standardized fashion; they screened patients daily for eligibility to undergo an SBT using the following criteria: awake, adequate cough, $\mathrm{PaO}_{2}>60 \mathrm{mmHg}, \mathrm{SpO}_{2} \geq 90 \%, \mathrm{FiO}_{2} \leq 0.4$, PEEP $\leq 10 \mathrm{~cm} \mathrm{H}_{2} \mathrm{O}$, respiratory rate $\leq 35 / \mathrm{min}$, minute ventilation $\leq 15$ $\mathrm{L} /$ minute, no inotropes/vasopressors, mean arterial pressure $>60 \mathrm{mmHg}$, and no evidence of myocardial ischemia. If all SBT criteria were met, patients underwent a 60-minute SBT, during which they breathed spontaneously through a T-tube circuit, a tracheotomy mask, or the ventilator circuit with continuous positive airway pressure of $5 \mathrm{~cm} \mathrm{H}_{2} \mathrm{O}$. Respiratory therapists could terminate the SBT if any of the following signs of failure persisted for more than 5 minutes: respiratory rate > $35 /$ min, oxygen saturation $<90 \%$, heart rate $>140 /$ min or a change in heart rate $\geq 20 \%$ in either direction, systolic 


\section{Anaesthesia \& Critical Care Medicine Journal}

blood pressure $<90$ or $>180 \mathrm{mmHg}$, or increased anxiety and diaphoresis. If the SBT was successful, respiratory therapists notified the ICU team for the purpose of prompt extubation. Weaning method and decisions to commence weaning and to extubate were at the discretion of the ICU team. When an SBT was not performed, or a patient was not extubated despite passing an SBT, respiratory therapists selected one of 8 reasons (agitation, procedure, physician request, worsening chest radiograph, mental status concerns, concern about airway patency, secretions, and inadequate cough or gag) or 'other' on the case report form .

\section{Results}

The frequency of screening for SBT eligibility was similar in the DI and control groups (92\% versus 93\% days) (Table 1). Among screened patients, the DI group were eligible for an SBT less often than the control group (32\% versus $36 \%$ days, $\mathrm{P}=0.003$ ). In both groups, respiratory therapists performed SBTs on $82 \%$ of days that SBT were indicated. Morning Sedation Agitation Scale (SAS) scores [6] was similar on days when SBTs were or were not done. The reasons for not performing an SBT when indicated differed between the groups. The most common reasons for missed SBTs in the DI group were patient agitation and concerns about airway patency. The most common reasons for not performing a SBTs in the control group were patient agitation, need for ICU procedures and physician request. The proportion of days patients passed an SBT but were not extubated was high and similar in the two groups (61\% DI versus $65 \%$ control). Morning SAS scores were similar between days when ventilator support was removed or not removed. The reasons for not extubating a patient on the day of a successful SBT differed between groups. In both groups, the most commonly cited reasons from the options provided on the case report form were concerns about mental status and physician request. In the control group, clinicians more often selected miscellaneous reasons ('other') which were not listed on the case report form as a reason for delayed extubation, compared with the interruption group. More than one third of patients in both groups were extubated without an SBT (39\% interruption versus $35 \%$ control, $\mathrm{P}=0.31$ ). The proportion of patients extubated after the first, second, or third successful SBT was similar between groups. Despite differences in SBT conduct, the median time to successful extubation was 7 days in both groups [5].

\section{Interpretation}

In this multicenter trial, screening for SBT eligibility was frequent, and SBTs were commonly performed when patients met screening criteria. However, patients were extubated less than $40 \%$ of the time on the day of a successful SBT. Patients in the DI group met SBT criteria on fewer days than the control group, possibly because clinicians perceived patients in the DI group to be less alert, as indicated by more concerns about 'mental status' and 'airway', despite similar SAS scores. These concerns may reflect the higher doses of opioids and benzodiazepines received by the DI group [5]. A limitation of our study is that while we recorded many reasons that SBTs and extubation were not done when indicated, not all were captured on the case report form. These findings are consistent with the lack of benefit of DI when a sedation protocol is used. SLEAP was a pragmatic trial; therefore our findings related to SBT and extubation practices likely reflect usual practice; and are similar to the $A B C$ trial in which $46 \%$ of patients were extubated on the day they first passed an SBT [4]. While screening and conduct of SBTs are easily protocolized, extubation decisions are challenging to protocolize as clinical judgment is necessary. Causes for delayed extubation once an SBT is passed represent an important yet understudied aspect of the process of liberation from mechanical ventilation.

\begin{tabular}{|c|c|c|c|}
\hline Variable & $\begin{array}{c}\text { Sedation protocol and daily } \\
\text { interruption } \mathbf{N = 2 1 4}\end{array}$ & $\begin{array}{c}\text { Sedation protocol } \\
\mathbf{N = 2 0 9}\end{array}$ & P value \\
\hline Total patient days & 2093 & 2468 & \\
\hline Days screen for SBT done & $1919(92)$ & $2305(93)$ & 0.03 \\
\hline Days criteria for SBT met & $615(32)$ & $836(37)$ & 0.003 \\
\hline Days SBT done when criteria met & $510(82)$ & $686(82)$ & 0.98 \\
\hline SAS at 8 AM before SBT, median (IQR) & $4(3,4)$ & $4(4,4)$ & 0.33 \\
\hline Days SBT not done when criteria met & $109(18)$ & $146(18)$ & 1.00 \\
\hline SAS at 8 AM, median (IQR) & $4(4,4)$ & $4(3,4)$ & 0.37 \\
\hline
\end{tabular}

Mehta S, et al. Spontaneous Breathing Trials in Patients Managed with a 


\section{Anaesthesia \& Critical Care Medicine Journal}

\begin{tabular}{|c|c|c|c|}
\hline Reasons SBT not done & & & 0.01 \\
\hline Agitation & $23(21)$ & $25(19)$ & \\
\hline Procedure & $6(6)$ & $14(11)$ & \\
\hline Physician request & $6(6)$ & $12(9)$ & \\
\hline Worsening chest radiograph & $2(2)$ & $7(5)$ & \\
\hline Mental status concerns & $9(8)$ & $4(3)$ & \\
\hline Concern about airway patency & $15(14)$ & $5(4)$ & \\
\hline Secretions & $7(7)$ & $11(9)$ & \\
\hline Inadequate cough or gag & $9(8)$ & $4(3)$ & \\
\hline Other & $31(29)$ & $47(36)$ & \\
\hline $\begin{array}{c}\text { Days patient extubated after passing } \\
\text { SBT }\end{array}$ & 139 (39) & $162(35)$ & 0.24 \\
\hline SAS at 8 AM before SBT, median (IQR) & $4(4,4)$ & $4(4,4)$ & 0.40 \\
\hline Days not extubated when passed SBT & $221(61)$ & $306(65)$ & 0.24 \\
\hline SAS at 8 am before SBT, median (IQR) & $4(3,4)$ & $4(4,4)$ & 0.07 \\
\hline $\begin{array}{l}\text { Reasons patient not extubated despite } \\
\text { passing SBT }\end{array}$ & & & 0.0001 \\
\hline Agitation & $8(4)$ & $4(2)$ & \\
\hline Procedure & $7(3)$ & $8(3)$ & \\
\hline Physician request & $26(12)$ & $28(11)$ & \\
\hline Worsening chest radiograph & $3(1)$ & $3(1)$ & \\
\hline Mental status concerns & $33(16)$ & $25(9)$ & \\
\hline Concern about airway patency & $23(11)$ & $8(3)$ & \\
\hline Secretions & $23(11)$ & $23(9)$ & \\
\hline Inadequate cough or gag & $8(4)$ & $6(2)$ & \\
\hline Other & $80(38)$ & $160(60)$ & \\
\hline Extubated without SBT & $84(39)$ & $72(35)$ & 0.31 \\
\hline Extubated after passing $1^{\text {st }} \mathrm{SBT}$ & $63(29)$ & $68(33)$ & 0.41 \\
\hline Extubated after passing $2^{\text {nd }}$ SBT & $39(18)$ & $31(15)$ & \\
\hline Extubated after passing $3^{\text {rd }}$ SBT & $8(4)$ & $14(7)$ & \\
\hline $\begin{array}{c}\text { Extubated after passing } 4^{\text {th }} \text { or later } \\
\text { SBT }\end{array}$ & $20(9)$ & $24(12)$ & \\
\hline
\end{tabular}

Table 1: Spontaneous breathing trials and extubation in both groups.

Data expressed as $\mathrm{n}(\%)$ unless otherwise indicated SBT=spontaneous breathing trial, SAS = sedation agitation scale

\section{Conflict of Interest}

The authors have no conflicts of interest to declare. 


\section{Anaesthesia \& Critical Care Medicine Journal}

\section{Statement of Contribution}

SM, LR conceived of the study; all authors were involved in design of the study; all authors have contributed to analysis and interpretation of the data and writing the article before submission.

\section{Sources of Support}

The SLEAP trial was funded by the Canadian Institutes of Health Research (CIHR). This secondary analysis of spontaneous breathing trials was funded by the Mount Sinai Hospital Department of Medicine.

\section{Acknowledgements}

L. Rose holds a Canadian Institutes for Health Research New Investigator Award. D. Cook is a Canada Research Chair of the Canadian Institutes for Health Research. We are grateful to the Canadian Critical Care Trials Group for their key role in this work. We are indebted to Andrew Sealy MD and Karen Bosma MD, for critical review of the manuscript.

\section{References}

1. Ely EW, Baker AM, Dunagan DP, Burke HL, Smith AC, et al. (1996) Effect on the duration of mechanical ventilation of identifying patients capable of breathing spontaneously. New Engl J Med 335(25): 1864-1869.

2. Ely EW, Baker AM, Evans GW, Haponik EF (1999) The prognostic significance of passing a daily screen of weaning parameters. Intensive Care Med 25(6): 581587.

3. Macintyre N (2013) The ventilator discontinuation process: an expanding evidence base. Respir Care 58(6): 1074-1086.

4. Girard T, Kress J, Fuchs B, Thomason J, Schweickert W, et al. (2008) Efficacy and safety of a paired sedation and ventilator weaning protocol for mechanically ventilated patients in intensive care (Awakening and Breathing Controlled trial): a randomised controlled trial. Lancet 371(9607): 126134.

5. Mehta S, Burry L, Cook D, Fergusson D, Steinberg M, et al. (2012) Daily sedation interruption in mechanically ventilated critically ill patients cared for with a sedation protocol: a randomized controlled trial. JAMA 308(19): 1985-1992.

6. Riker RR, Picard JT, Fraser GL (1999) Prospective evaluation of the Sedation-Agitation Scale for adult critically ill patients. Crit Care Med 27(7): 1325-1329.

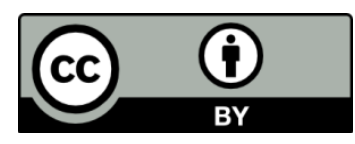

Mehta S, et al. Spontaneous Breathing Trials in Patients Managed with a Sedation Protocol or a Sedation Protocol with Daily Interruption. Anaesth Critic Care Med J 2016, 1(2): 000110. 